\title{
Minimal change nephrotic syndrome. Histopathology and steroid-responsiveness
}

Children with minimal change nephrotic syndrome (MCNS) generally respond promptly to initial steroid therapy, undergo a complete remission, and have a good prognosis. MCNS needs to be separated therefore, from other forms of the nephrotic syndrome in which children fail to respond promptly. Investigations carried out by the International Study of Kidney Disease in Children (ISKDC) have confirmed the generally held belief that nephrotic children who respond during an 8-week course of intensive corticosteroid therapy are likely to have MCNS. ${ }^{1}$ That likelihood is of the order of $90 \%$. Children who do not respond, particularly those aged 6 years or older, are likely to have other glomerular diseases, but renal biopsy in nonresponders (continued proteinuria after 8 weeks of treatment) shows that about one-quarter of all non-responders and half of those younger than 6 years none the less have MCNS. ${ }^{1}$

It has been essential therefore, to identify the histopathological criteria of 'minimal change' and to recognise the limits of histopathological variability. Minor variability within the histopathological category of MCNS was recognised by the ISKDC at the outset, ${ }^{2}$ and a recent report ${ }^{3}$ contains an analysis of clinical characteristics and responses to treatment in children having histopathological subcategories of MCNS. Such subcategories include nil disease, focal glomerular obsolescence, mild mesangial thickening, mild mesangial hypercellularity, and focal tubular changes. The criteria allow MCNS to be separated from both focal segmental glomerular sclerosis and more severe degrees of mesangial proliferation. A category of diffuse mesangial hypercellularity has been recognised as intermediate between mild mesangial hypercellularity and diffuse proliferative glomerulonephritis. These definitions and criteria have recently been defined even more rigorously and incorporated into the World Health Organisation's classification of glomerular diseases. ${ }^{4}$

The ISKDC study of clinical characteristics at onset showed no important differences among the histopathological subcategories of $\mathrm{MCNS}^{3}{ }^{3}$ except that young patients tended to have glomerular obsolescence, a phenomenon well known to paediatric pathologists. However, more children having mild mesangial hypercellularity and focal tubular changes than those having nil disease, focal glomerular obsolescence, or mild mesangial thickening failed to respond promptly to corticosteroid therapy. Children with diffuse mesangial hypercellularity, that is with a degree of mesangial hypercellularity exceeding the limit set for MCNS, also had a significantly poorer rate of steroid responsiveness than did children with MCNS.

The differences at 8 weeks disappeared however, during 52 weeks of continued corticosteroid therapy. In other words, children with mild mesangial hypercellularity, focal tubular changes, and diffuse mesangial hypercellularity experienced delayed remissions, perhaps resulting from, perhaps abetted by, the prolonged treatment. The ultimately good responsiveness in all categories of MCNS and in diffuse mesangial hypercellularity and the paucity of differentiating clinical characteristics suggests that the differences in histology reflect different severities of glomerular injury rather than different disease processes. Perhaps mild mesangial hypercellularity reflects a more severe degree of glomerular injury than does nil disease and diffuse mesangial hypercellularity is a more severe injury than mild mesangial hypercellularity. On those grounds alone, the response to therapy might be slower or poorer, and recognising different degrees of hypercellularity might resolve the discrepancies reported in responsiveness of nephrotic children with mesangial proliferative abnormalities. ${ }^{5-9}$ The significance of focal tubular changes is unknown.

The ISKDC analysis of late responses to steroid therapy in patients with different subcategories of MCNS and with diffuse mesangial hypercellularity showed no significant differences. ${ }^{3}$ There were no differences among the rates of early relapse (relapse during the first 8 weeks of treatment), frequent relapse ( 2 or more relapses within 6 months of the initial response), late non-response (failure to respond to steroid therapy after having responded previously), and death. Those observations, particularly those relating to late non-response, can be interpreted to mean that relapses are not necessarily of the same severity as the initial episode. We would predict, if relapses were unequal in the severity of glomerular damage, that late non-responsiveness 
would be distributed randomly among the different histopathological categories, which is precisely what the data show. Furthermore, the frequency of relapses in individual patients was inconstant and lacked correlation with the histopathological findings. ${ }^{10}$

The ISKDC data have bearing therefore, on our interpretation of the natural history of the idiopathic nephrotic syndrome in childhood. An initially severe lesion might well be associated with non-responsiveness or with delayed responsiveness to corticosteroid therapy and might carry a high risk of glomerular scarring. ${ }^{11}$ It would be wrong however, to assume that late non-responsiveness or the late development of focal segmental glomerular sclerosis after an interval of several years necessarily relates to the severity of the initial glomerular disease. Relapses may continue to follow the same pattern of response after the first attack, as they do in the majority of patients, but there is no requirement that they do so. Relapses in that view are not smouldering embers, left over from the initial conflagration, but new outbursts, presumably triggered by identical or similar mechanisms.

Unanswered is the question of steroid-dependence in relation to glomerular histopathology. If steroiddependent patients include a fairly high proportion with mesangial hypercellularity and glomerular sclerosis ${ }^{12}$ steroid-dependence would seem to relate to the severity of glomerular disease. An alternative view however, has been expressed by Arant et al., ${ }^{13}$ who suggested that steroid-dependence is related to functional adrenal insufficiency as the result of steroid-induced adrenal suppression. It is also conceivable that some of the glomerular alterations in patients receiving long-term corticosteroid therapy are related to the treatment rather than to the disease, as experimental studies have shown retention of macromolecules in cortisone-treated animals. ${ }^{14}$ These altered mesangial kinetics might explain the mesangial accumulation of IgM in some patients ${ }^{5} 15$ and the fairly frequent electron microscopical demonstration of electron-dense 'deposits' in children with longstanding MCNS. ${ }^{16}$

It appears that early response to steroid therapy in the idiopathic nephrotic syndrome predicts the subsequent course with an accuracy of about $85 \%$. The development of late non-response in $15 \%$ however, injects a small element of doubt into the physician's prognostication. Such patients might be candidates for treatment with immunosuppressant drugs, and a large number of them will do well in the long run. A small number of patients may however, develop progressive renal insufficiency as the result of renewed attacks of glomerular injury.

\section{References}

1 A Report of the International Study of Kidney Disease in Children. The primary nephrotic syndrome in children. Identification of patients with minimal change nephrotic syndrome from initial response to prednisone. $J$ Pediatr $1981 ; 98: 561-4$.

2 Churg J, Habib R, White R H R. Pathology of the nephrotic syndrome in children. A Report for the International Study of Kidney Disease in Children. Lancet 1970 ; i: 1299-302.

3 A Report of the International Study of Kidney Disease in Children. Primary nephrotic syndrome in children: clinical significance of histopathologic variants of minimal change and of diffuse mesangial hypercellularity. Kidney Int 1981; 20: 765-71.

4 Churg J, Sobin L H, eds. Renal diseases. Classification and atlas of glomerular diseases. New York-Tokyo: Igaku-Shoin, 1982:67-9.

5 Bhasin H K, Abuelo J G, Nayak R, Esparza A R. Mesangial proliferative glomerulonephritis. Lab Invest $1978 ; 39: 21-9$.

- Brown E A, Upadhyaya K, Hayslett J P, Kashgarian M, Siegel N J. The clinical course of mesangial proliferative glomerulonephritis. Medicine 1979; 58: 295-303.

7 Murphy W M, Jukkola A F, Roy S, III. Nephrotic syndrome with mesangial-cell proliferation in children-a distinct entity? Am J Clin Pathol 1979; 72: 42-7.

8 Waldherr R, Gubler M C, Levy M, Broyer M, Habib R. The significance of pure diffuse mesangial proliferation in idiopathic nephrotic syndrome. Clin Nephrol 1978; 10: $171-9$.

9 Allen W R, Travis L B, Cavallo T, Brouhard B H, Cunningham R J, III. Immune deposits and mesangial hypercellularity in minimal change nephrotic syndrome: clinical relevance. J Pediatr 1982; 100: 188-91.

10 A Report of the International Study of Kidney Disease in Children. Early identification of frequent relapses among children with minimal change nephrotic syndrome. $J$ Pediatr 1982;101 : in press.

11 Rosen S, Galvanek E, Levy M, Habib R. Glomerular disease. Hum Pathol 1981; 12: 964-77.

12 Siegel N J, Gaudio K M, Krassner L S, McDonald B M, Anderson F P, Kashgarian $M$. Steroid-dependent nephrotic syndrome in children: histopathology and relapses after cyclophosphamide treatment. Kidney Int 1981 ; 19: 454-9.

13 Arant B S, Jr, Singer S A, Bernstein J. Steroid-dependent nephrotic syndrome. J Pediatr 1982; 100: 328-33.

14 Haakenstad A O, Case J B, Mannik M. Effect of cortisone on the disappearance kinetics and tissue localization of soluble immune complexes. J Immunol 1975; 114: 1153-60.

15 Cohen A H, Border W A, Glassock R J. Nephrotic syndrome with glomerular mesangial IgM deposits. Lab Invest 1978; 38: 610-9.

16 Gubler M C, Kleinknecht C, Behrouz-Agdam A, Habib R. Clinicopathological correlations and ultrastructural study in children presenting with a corticosteroid resistant lipoid nephrosis (abstract). In the proceedings of the Second International Symposium on Pediatric Nephrology, Washington 1974: 24.

JAY Bernstein, JR Department of Anatomic Pathology, William Beaumont Hospital, Royal Oak, Michigan 48072, USA Chester M EdelmanN, JR Albert Einstein College of Medicine, Bronx, NY 10461, USA 